\title{
A NOVEL PROCEDURE TO ASSESS THE NON-ENZYMATIC HYDROGEN-PEROXIDE ANTIOXIDANT CAPACITY OF METABOLITES WITH HIGH UV ABSORPTION
} SHORT COMMUNICATION

\author{
KRISTÓF CSEPREgI and ÉVA HideG* \\ Department of Plant Biology, Institute of Biology, University of Pécs, \\ Ifjúság u. 6, H-7624 Pécs, Hungary
}

\begin{abstract}
Assays assessing non-enzymatic hydrogen peroxide antioxidant capacities are often hampered by the high UV absorption of the sample itself. This is a typical problem in studies using plant extracts with high polyphenol content. Our assay is based on comparing the $405 \mathrm{~nm}$ absorption of the product of potassium iodine and hydrogen peroxide in the presence and absence of a putative hydrogen peroxide reactive antioxidant. This method is free of interference with either hydrogen peroxide or antioxidant self-absorption and it is also suitable for high-throughput plate reader applications.
\end{abstract}

Keywords: Antioxidant assay - hydrogen peroxide - polyphenol - UV absorption

Hydrogen peroxide is a Janus-faced reactive oxygen species (ROS). Depending on its cellular localization and concentration $\mathrm{H}_{2} \mathrm{O}_{2}$ is capable of causing or spreading oxidative damage, and it is also an important signal molecule evoking developmental or stress responses [1]. Consequently, regulation of cellular $\mathrm{H}_{2} \mathrm{O}_{2}$ concentrations by antioxidants is an important aspect in plants [2] as well as in other life forms [1]. In addition to $\mathrm{H}_{2} \mathrm{O}_{2}$ specific peroxidases, there are several metabolites which may act as non-enzymatic $\mathrm{H}_{2} \mathrm{O}_{2}$ antioxidants. Examples of $\mathrm{H}_{2} \mathrm{O}_{2}$ neutralizing compounds in plants include ascorbic acid, glutathione [3] and phenolic compounds [4]. Similarly to enzyme activity assays, methods are also available to assess non-enzymatic antioxidant capacities. Specific $\mathrm{H}_{2} \mathrm{O}_{2}$ neutralizing capacities are based on following either changes in $\mathrm{H}_{2} \mathrm{O}_{2}$ concentrations either directly at $230 \mathrm{~nm}$ [5] or using $\mathrm{H}_{2} \mathrm{O}_{2}$ oxidizable probes, such as 4-aminoantipyrine [6]. The former method is difficult to apply in studies involving phenolic rich plant extracts which have high UV absorption themselves. The second method is free from this issue; however, the reaction with the applied probe with $\mathrm{H}_{2} \mathrm{O}_{2}$ to yield the coloured quinoneimine dye requires horseradish peroxidase which is to be added to the assay [7]. The problem with

*Corresponding author; e-mail address: ehideg@gamma.ttk.pte.hu

Abbreviations: Quercetin - 2-(3,4-dihydroxyphenyl)-3,5,7-trihydroxy-4H-chromen-4-one; ROS reactive oxygen species; Trolox - 6-hydroxy-2,5,7,8-tetramethyl-chroman-2-carboxylic acid; UV - ultraviolet light. 

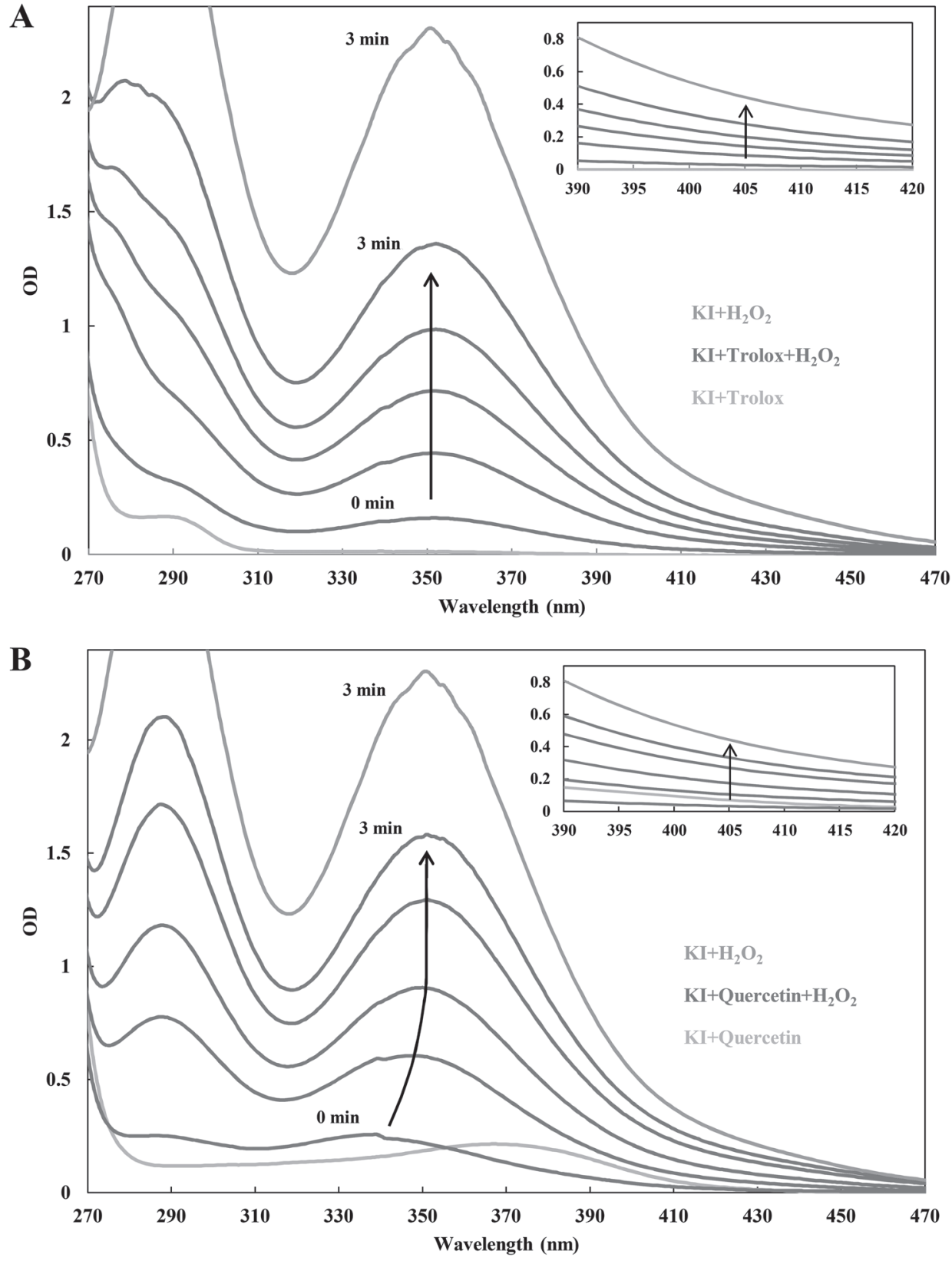

Fig. 1. Absorption spectra of iodine ions from the oxidation of $850 \mathrm{mM} \mathrm{KI}$ by $1 \mathrm{mM} \mathrm{H}_{2} \mathrm{O}_{2}$ in 1:10 ethanol: potassium-phosphate $(100 \mathrm{mM}, \mathrm{pH} 7.0)$. Blue line: after 3 min incubation in the absence of antioxidant. Red lines: after $0,1,1.5,2$, and $3 \mathrm{~min}$ incubation in the presence of $300 \mu \mathrm{M}$ Trolox (A); and 0, 0.5, 1, 2, $3 \mathrm{~min}$ incubation in the presence of $30 \mu \mathrm{M}$ quercetin (B) as antioxidants. Green lines show absorption spectra of the antioxidants only 
enzyme mediated protocols is that organic solvents which are often required for extracting polyphenols from plant derived samples may inhibit the peroxidase catalyst of the colour reaction. Consequently, the 4-aminoantipyrine method cannot be applied in these studies and a similar restriction applies to protocols based on other $\mathrm{H}_{2} \mathrm{O}_{2}+$ peroxidase + chromophore systems.

Plant polyphenols are special metabolites with a variety of functions including ROS neutralizing. These are contained in photosynthetic tissues as well as in berries and other foodstuff. Consequently, antioxidant capacities are of special interest not only for plant stress physiology but also for food science studies. The aim of the present work was to develop a technique for the assessment of $\mathrm{H}_{2} \mathrm{O}_{2}$ neutralizing capacity of phenolic rich samples; which (i) is easy and cost-effective to perform, (ii) does not involve $\triangle \mathrm{OD}$ measurements in the UV spectral region and (iii) does not require the presence of peroxidase enzymes.

Our assay is based on oxidation of potassium iodine (KI) by $\mathrm{H}_{2} \mathrm{O}_{2}$, but instead of the maximum absorption of the iodine product $\left(\mathrm{I}_{2}\right)$ at $350-360 \mathrm{~nm}$ [8] the reaction is followed at $405 \mathrm{~nm}$. As shown in Fig. 1, absorption of the chromophore at this wavelength is not modified by the presence of polyphenols.

Figure 1A illustrates the functioning of the assay using Trolox, a synthetic vitamin $\mathrm{E}$ analogue which is frequently applied as reference compound in a variety of in antioxidant assays. When present at $300 \mu \mathrm{M}$ concentration, Trolox did not fully prevent the oxidation of $\mathrm{KI}$ by $\mathrm{H}_{2} \mathrm{O}_{2}$; as shown by increasing absorption of the oxidised product (red spectrums connected by the arrow in Fig. 1A). Trolox itself does not absorb at wavelengths higher than $300 \mathrm{~nm}$ (green line in Fig. 1A), consequently its presence does not change the shape of chromophore absorption spectrum. After 3 min absorption at both $350 \mathrm{~nm}$ and $405 \mathrm{~nm}$ was $40 \%$ lower in the presence of Trolox (the highest spectrum drawn with red line) than in the absence of the antioxidant (blue line, Fig. 1A). This figure also illustrates the functioning of the assay, the $\mathrm{H}_{2} \mathrm{O}_{2}$ antioxidant capacity of a compound is characterized by the difference in chromophore absorption in the absence and in the presence of the antioxidant containing sample recorded after the same incubation time. The higher the $\mathrm{H}_{2} \mathrm{O}_{2}$ reactivity, the larger the difference between the two absorptions. In this way, the antioxidant capacity of a biological sample or any other putative antioxidant can be given relative to that of a test compound, for example Trolox.

Figure 1B shows the same experiment carried out using quercetin instead of Trolox. Quercetin is a flavonoid, one of the most abundant dietary flavonols present in plants in various glycosylated forms [4] with assumed health benefits [9]. Quercetin itself absorbs UV light, and its $370 \mathrm{~nm}$ absorption maximum (green line in Fig. 1B) overlaps with the absorption of the coloured product of $\mathrm{KI}$ oxidation by $\mathrm{H}_{2} \mathrm{O}_{2}$. As a result, the absorption maximum of a $\mathrm{KI}+$ quercetin $+\mathrm{H}_{2} \mathrm{O}_{2}$ sample is shifting as the oxidation reaction (of both KI and quercetin) progresses in time (Fig. 1B). This spectral shift does not affect absorption at $405 \mathrm{~nm}$, as shown in the inset in Fig. 1B, making absorption at this higher wavelength a more reliably indicator of KI oxidation. When assessing the antioxidant capacities of a large number of samples, which is often the case in both plant stress and food science applications, following full 
absorption spectra to establish whether shifting of spectral maxima due to the selfabsorption of samples interferes with the UV absorption of the chromophore would be too time consuming. Reading absorptions at a single wavelength after a set incubation time provides the possibility of fast measurements.

In summary, the above comparison shows the advantage of choosing a wavelength which does not interfere with the UV absorption of polyphenols. Choosing $405 \mathrm{~nm}$ is a good compromise, because with increasing the KI concentration in the assay, $\triangle \mathrm{OD}$ $405 \mathrm{~nm}$ is still high enough for analysis. Also, this wavelength is included as preset factory setup in most plate readers allowing a as high-throughput assay to be based on this protocol.

\section{ACKNOWLEDGEMENT}

Supported by the Hungarian Scientific Grant Agency (grant number OTKA K112309).

\section{REFERENCES}

1. Halliwell, B., Gutteridge, J. M. C. (2006) Free Radicals in Biology and Medicine. Clarendon Press, Oxford.

2. Neill, S. J., Desikan, R., Clarke, A., Hurst, R., Hancock, J. (2002) Hydrogen peroxide and nitric oxide as signalling molecules in plants. J. Exp. Bot. 53, 1237-1247.

3. Noctor, G., Foyer, C. H. (1998) Ascorbate and glutathione: keeping active oxygen under control. Annu. Rev. Plant Phys. 49, 249-279.

4. Kähkönen, M. P., Hopia, I. A., Vuorela, J. H., Rauha, J.-P., Pihlaja, K., Kujala, S. T., Heinonen, M. (1999) Antioxidant activity of plant extracts containing phenolic compounds. J. Agric. Food Chem. 47, 3954-3962.

5. Keser, S., Celik, S., Turkoglu, S., Yilmaz, Ö., Turkoglu, I. (2012) Hydrogen peroxide radical scavenging and total antioxidant activity of hawthorn. Chem. J. 2, 9-12.

6. Fernando, C. D., Soysa, P. (2015) Optimized enzymatic colorimetric assay for determination of hydrogen peroxide $\left(\mathrm{H}_{2} \mathrm{O}_{2}\right)$ scavenging activity of plant extracts. Methods X. 2, 283-291.

7. Shah, V., Baldrian, P., Eichlerova, I., Dave, R., Madamwar, D., Nerud, F., Gross, R. (2006) Influence of dimethyl sulfoxide on extracellular enzyme production by Pleurotus ostreatus. Biotechnol. Lett. 28, 651-655.

8. Junglee, S., Urban, L., Sallanon, H., Lopez-Lauri, F. (2014) Optimized assay for hydrogen peroxide determination in plant tissue using potassium iodide. American J. Anal. Chem. 5, 730-736.

9. Formica, J. V., Regelson, W. (1995) Review of the biology of quercetin and related bioflavonoids. Food Chem. Toxicol. 33, 1061-1080. 